\title{
Some properties for $\alpha$-starlike functions with respect to $k$-symmetric points of complex order
}

\begin{abstract}
In the present work, we introduce the subclass $\mathcal{T}_{\gamma, \alpha}^{k}(\varphi)$, of starlike functions with respect to $k$-symmetric points of complex order $\gamma(\gamma \neq 0)$ in the open unit disc $\triangle$. Some interesting subordination criteria, inclusion relations and the integral representation for functions belonging to this class are provided. The results obtained generalize some known results, and some other new results are obtained.
\end{abstract}

1. Introduction. Let $\mathcal{A}$ be the class of functions $f(z)$ of the form:

$$
f(z)=z+\sum_{k=2}^{\infty} a_{k} z^{k},
$$

which are analytic in the open unit disk $\triangle=\{z: z \in \mathbb{C},|z|<1\}$. A function $f \in \mathcal{A}$ is subordinate to an univalent function $g \in \mathcal{A}$, written $f(z) \prec g(z)$, if $f(0)=g(0)$ and $f(\triangle) \subseteq g(\triangle)$. Let $\Omega$ be the family of analytic functions $w(z)$ in the unit disc $\triangle$ satisfying the conditions $w(0)=0$ and $|w(z)|<1$, for $z \in \triangle$. Note that $f(z) \prec g(z)$ if there is a function $w(z) \in \Omega$ such that $f(z)=g(w(z))$. Further, let $\wp$ be the class of analytic functions $p$ with $p(0)=1$, which are convex and univalent in $\triangle$ and satisfy

2010 Mathematics Subject Classification. 30C45.

Key words and phrases. Analytic functions, starlike functions of complex order, convex functions of complex order, $\alpha$-starlike, $k$-symmetric points, subordination. 
the condition

$$
\operatorname{Re}\{p(z)\}>0, \quad(z \in \triangle) .
$$

For a given positive integer $k$, let $\varepsilon=\exp (2 \pi i / k)$ and

$$
f_{k}(z)=\frac{1}{k} \sum_{v=0}^{k-1} \varepsilon^{-v} f\left(\varepsilon^{v} z\right), \quad(z \in \triangle) .
$$

Let $\varphi(z) \in \wp$, we denote by $\mathcal{S}_{s}^{k}(\varphi), \mathcal{C}_{s}^{k}(\varphi)$ and $\mathcal{T}_{\alpha}^{k}(\varphi)$ the familiar subclasses of $\mathcal{A}$ consisting of starlike, convex and $\alpha$-starlike functions with respect to $k$-symmetric points in $\triangle$ respectively. That is

$$
\begin{aligned}
& \mathcal{S}_{s}^{k}(\varphi)=\left\{f \in \mathcal{A}: \frac{z f^{\prime}(z)}{f_{k}(z)} \prec \varphi(z) \quad(z \in \triangle)\right\}, \\
& \mathcal{C}_{s}^{k}(\varphi)=\left\{f \in \mathcal{A}: \frac{\left(z f^{\prime}(z)\right)^{\prime}}{f_{k}(z)} \prec \varphi(z) \quad(z \in \triangle)\right\},
\end{aligned}
$$

and

$$
\mathcal{T}_{\alpha}^{k}(\varphi)=\left\{f \in \mathcal{A}: \frac{\alpha z\left(z f^{\prime}(z)\right)^{\prime}+(1-\alpha) z f^{\prime}(z)}{\alpha z f_{k}^{\prime}(z)+(1-\alpha) f_{k}(z)} \prec \varphi(z) \quad(z \in \triangle)\right\} .
$$

The class $\mathcal{T}_{\alpha}^{k}(\varphi)$ was introduced and studied by Parvatham and Radha [11] and this class generalizes the classes defined by Pascu [12], Das and Singh [5] as well as the classes $\mathcal{S}_{s}^{k}(\varphi)$ and $\mathcal{C}_{s}^{k}(\varphi)$ which were studied recently by Wang et al. [18]. Recently several subclasses of analytic functions with respect to $k$-symmetric points were introduced and studied by various authors (see [1], [2], [14], [18], [19], [21]). Following Ma and Minda [7], Ravichandran et al. [15] defined a more general class related to the class of starlike functions of complex order as follows.

A function $f(z) \in \mathcal{A}$ is said to be in the class $\mathcal{S}_{\gamma}(\varphi)$, if it satisfies the following subordination condition

$$
1+\frac{1}{\gamma}\left(\frac{z f^{\prime}(z)}{f(z)}-1\right) \prec \varphi(z) \quad\left(z \in \triangle, \gamma \in \mathbb{C}^{*}=\mathbb{C} \backslash\{0\} \text { and } \varphi(z) \in \wp\right) .
$$

Furthermore, a function $f(z) \in \mathcal{A}$ is said to be in the class $\mathcal{C}_{\gamma}(\varphi)$, if it also satisfies the subordination condition

$$
1+\frac{1}{\gamma}\left(\frac{z f^{\prime \prime}(z)}{f^{\prime}(z)}\right) \prec \varphi(z) \quad\left(z \in \triangle, \gamma \in \mathbb{C}^{*}=\mathbb{C} \backslash\{0\} \text { and } \varphi(z) \in \wp\right) .
$$

Motivated by the classes $\mathcal{T}_{\alpha}^{k}(\varphi), \mathcal{S}_{\gamma}(\varphi)$ and $\mathcal{C}_{\gamma}(\varphi)$, we now introduce and investigate the following subclasses of $\mathcal{A}$, and obtain some interesting results.

Moreover, for some non-zero complex number $\gamma$, we consider the subclasses $\mathcal{T}_{\gamma, \alpha}^{k}(\varphi), \mathcal{F}_{\gamma, \alpha}^{k}(\varphi)$ of $\mathcal{A}$ as follows. 
Definition 1. Let $\mathcal{T}_{\gamma, \alpha}^{k}(\varphi)$ denote the class of functions $f$ in $\mathcal{A}$ satisfying the following condition

$$
1+\frac{1}{\gamma}\left(\frac{\alpha z\left(z f^{\prime}(z)\right)^{\prime}+(1-\alpha) z f^{\prime}(z)}{\alpha z f_{k}^{\prime}(z)+(1-\alpha) f_{k}(z)}-1\right) \prec \varphi(z),
$$

where $\varphi(z) \in \wp$ and $\alpha \geq 0$.

Definition 2. Let $\mathcal{F}_{\gamma, \alpha}^{k}(\varphi)$ denote the class of functions $f \in \mathcal{A}$ satisfying the subordination condition

$$
1+\frac{1}{\gamma}\left(\frac{\alpha z\left(z f^{\prime}(z)\right)^{\prime}+(1-\alpha) z f^{\prime}(z)}{\alpha z \xi_{k}^{\prime}(z)+(1-\alpha) \xi_{k}(z)}-1\right) \prec \varphi(z),
$$

where $\xi_{k}(z)=\frac{1}{k} \sum_{v=0}^{k-1} \varepsilon^{-v} \xi\left(\varepsilon^{v} z\right), \xi(z) \in \mathcal{T}_{\gamma, \alpha}^{k}(\varphi), \varphi(z) \in \wp$, and $\alpha \geq 0$.

By giving specific values to the parameters $k, \gamma$ and $\alpha$ in the class $\mathcal{T}_{\gamma, \alpha}^{k}(\varphi)$, we get the following new subclass of analytic functions.

Remark 1. Putting $k=1$, we obtain the following definition.

Definition 3. Let $\mathcal{T}_{\gamma, \alpha}(\varphi)$ denote the class of functions $f \in \mathcal{A}$ satisfying the following condition

$$
1+\frac{1}{\gamma}\left(\frac{\alpha z\left(z f^{\prime}(z)\right)^{\prime}+(1-\alpha) z f^{\prime}(z)}{\alpha z f^{\prime}(z)+(1-\alpha) f(z)}-1\right) \prec \varphi(z),
$$

where $\varphi(z) \in \wp$ and $\alpha \geq 0$.

Remark 2. If we set $\alpha=0$, we have the following definition.

Definition 4. Let $\mathcal{S}_{\gamma}^{k}(\varphi)$ denote the class of functions $f$ in $\mathcal{A}$ satisfying the following condition

$$
1+\frac{1}{\gamma}\left(\frac{z f^{\prime}(z)}{f_{k}(z)}-1\right) \prec \varphi(z)
$$

where $\varphi(z) \in \wp$.

\section{Remark 3.}

(i) $\mathcal{T}_{1, \alpha}^{k}(\varphi)=K_{k}(\alpha, \varphi)$ (see Paravatham and Radha [11]), where $K_{k}(\alpha, \varphi)$ is the class of $\alpha$-starlike functions with respect to $k$ symmetric points.

(ii) $\mathcal{T}_{\gamma, \alpha}^{1}\left(\frac{1+(1-2 \beta) z}{1-z}\right)=\mathcal{S C}(\gamma, \alpha, \beta)(0 \leq \beta<1)$ (see Altintas et al. [3]).

(iii) $\mathcal{T}_{1,0}^{k}(\varphi)=\mathcal{S}_{s}^{k}(\varphi)$ and $\mathcal{T}_{1,1}^{k}(\varphi)=\mathcal{C}_{s}^{k}(\varphi)$ (see Wang et al. [18]).

(iv) $\mathcal{T}_{\gamma, 0}^{1}(\varphi)=\mathcal{S}_{\gamma}(\varphi)$ (see Ravichandran et al. [15]).

(v) $\mathcal{T}_{1, \alpha}^{1}\left(\frac{1-z}{1+z}\right)=K(\alpha)$ (see Pascu and Podaru [13]), where $K(\alpha)$ is the class of $\alpha$-starlike functions.

(vi) $\mathcal{T}_{1,1}^{2}\left(\frac{1-z}{1+z}\right)=\mathcal{C}_{s}$ (see Das and Singh [5]), where $\mathcal{C}_{s}$ is the class of convex functions with respect to symmetric points.

(vii) $\mathcal{T}_{1,0}^{2}\left(\frac{1-z}{1+z}\right)=\mathcal{S}_{s}$ (see Sakaguchi [17]), where $\mathcal{S}_{s}$ is the class of starlike functions with respect to symmetric points. 
(viii) $\mathcal{T}_{1-\beta, 0}^{k}\left(\frac{1-z}{1+z}\right)=\mathcal{S}_{s}^{*, k}(\beta)$ and $\mathcal{T}_{1-\beta, 1}^{k}\left(\frac{1-z}{1+z}\right)=\mathcal{C}_{s}^{k}(\beta)(0 \leq \beta<1)$ (see Chand and Singh [4]), where $\mathcal{S}_{s}^{*, k}(\beta)$ is the class of starlike functions with respect to $k$-symmetrical points of order $\beta$ and $\mathcal{C}_{s}^{k}(\beta)$ is the class of convex functions with respect to $k$-symmetrical points of order $\beta$.

(viiii) $\mathcal{T}_{\gamma, 0}^{1}\left(\frac{1-z}{1+z}\right)=\mathcal{S}_{\gamma}$ (see Nasr and Aouf [9]), where $\mathcal{S}_{\gamma}$ is the class of starlike functions of complex order.

(x) $\mathcal{T}_{\gamma, 1}^{1}\left(\frac{1-z}{1+z}\right)=\mathcal{C}_{\gamma}$ (see Nasr and Aouf [8]) and Wiatrowski [20] where $\mathcal{C}_{\gamma}$ is the class of convex functions of complex order.

(xi) $\mathcal{T}_{1-\alpha, 0}^{1}\left(\frac{1-z}{1+z}\right)=\mathcal{S}(\alpha)$ and $\mathcal{T}_{1-\alpha, 1}^{1}\left(\frac{1-z}{1+z}\right)=\mathcal{C}(\alpha)$ (see Robertson [16]), where $\mathcal{S}(\alpha)$ is the class of starlike functions of order $\alpha(0 \leq \alpha<1)$ and $\mathcal{C}(\alpha)$ is the class of convex functions of order $\alpha(0 \leq \alpha<1)$.

To prove our main results, we need the following lemmas.

Lemma $1([10])$. Let $\mathbb{k}, \vartheta$ be complex numbers. Suppose that $h(z)$ is convex and univalent in $\triangle$ with

$$
h(0)=1 \quad \text { and } \quad \operatorname{Re}[\mathbb{k} h(z)+\vartheta]>0 \quad(z \in \triangle),
$$

and let $q(z)$ be analytic in $\triangle$ with $q(0)=1$ and $q(z) \prec h(z)$.

If $p(z)=1+p_{1} z+p_{2} z^{2}+\cdots \in \wp$ with $p(0)=1$, then

$$
p(z)+\frac{z p^{\prime}(z)}{\mathbb{k} q(z)+\vartheta} \prec h(z)
$$

implies that $p(z) \prec h(z)$.

Lemma 2 (see $[6,7])$. Let $\mathbb{k}, \vartheta$ be complex numbers. Suppose that $h(z)$ is convex and univalent in $\triangle$ and satisfies (1.8). If $p(z)=1+p_{1} z+p_{2} z^{2}+\ldots$ $\in \wp$ and satisfies the subordination

$$
p(z)+\frac{z p^{\prime}(z)}{\mathbb{k} p(z)+\vartheta} \prec h(z),
$$

then

$$
p(z) \prec h(z) .
$$

2. Main result. Unless otherwise mentioned, we assume throughout this article that $f \in \mathcal{A}, \alpha>0, \varphi \in \wp$ and $\gamma \in \mathbb{C}^{*}$.

Proposition 1. Let $f \in \mathcal{T}_{\gamma, \alpha}(\varphi)$ and $\operatorname{Re} \frac{1}{\alpha}[\alpha \gamma(\varphi(z)-1)+1]>0$, then $f \in \mathcal{S}_{\gamma}(\varphi)$.

Proof. Set

$$
p(z)=1+\frac{1}{\gamma}\left(\frac{z f^{\prime}(z)}{f(z)}-1\right)
$$


then $p$ is an analytic function with $p(0)=1$. By differentiating (2.1) logarithmically, we get

$$
\begin{aligned}
1+\frac{1}{\gamma} & \left(\frac{\alpha z\left(z f^{\prime}(z)\right)^{\prime}+(1-\alpha) z f^{\prime}(z)}{\alpha z f^{\prime}(z)+(1-\alpha) f(z)}-1\right) \\
& =p(z)+\frac{\alpha z p^{\prime}(z)}{1+\alpha \gamma[p(z)-1]} \prec \varphi(z), \quad(z \in \triangle) .
\end{aligned}
$$

The conclusion of Proposition 1 yields from Lemma 2 , by taking $\mathbb{k}=\gamma$ and $\vartheta=\frac{1-\alpha \gamma}{\alpha}$.

Similarly, we can prove the following proposition.

Proposition 2. Let $\operatorname{Re} \frac{1}{\alpha}[\alpha \gamma(\varphi(z)-1)+1]>0$. Then

$$
F(z)=I_{\alpha}(f)=\frac{1}{\alpha z^{(1 / \alpha)-1}} \int_{0}^{z} t^{(1 / \alpha)-2} f(t) d t \in \mathcal{S}_{\gamma}(\varphi)
$$

whenever $f(z) \in \mathcal{S}_{\gamma}(\varphi)$.

Theorem 1. Let $f \in \mathcal{T}_{\gamma, \alpha}^{k}(\varphi)$ and $\operatorname{Re} \frac{1}{\alpha}[1+\alpha \gamma(\varphi(z)-1)]>0$. Then $f_{k}$ defined by (1.2) is in $\mathcal{T}_{\gamma, \alpha}(\varphi)$. Further, we have $f_{k} \in \mathcal{S}_{\gamma}(\varphi)$.

Proof. Let $f \in \mathcal{T}_{\gamma, \alpha}^{k}(\varphi)$. Replacing $z$ by $\varepsilon^{\mu} z\left(\mu=0,1, \ldots, k-1 ; \varepsilon^{k}=1\right)$ in (1.4), then (1.4) also holds true, that is,

$$
1+\frac{1}{\gamma}\left(\frac{(1-\alpha) \varepsilon^{\mu} z f^{\prime}\left(\varepsilon^{\mu} z\right)+\alpha \varepsilon^{\mu} z\left[f^{\prime}\left(\varepsilon^{\mu} z\right)+\varepsilon^{\mu} z f^{\prime \prime}\left(\varepsilon^{\mu} z\right)\right]}{(1-\alpha) f_{k}\left(\varepsilon^{\mu} z\right)+\alpha \varepsilon^{\mu} z f_{k}^{\prime}\left(\varepsilon^{\mu} z\right)}-1\right) \prec \varphi(z) .
$$

According to the definition of $f_{k}$ and $\varepsilon^{k}=1$, we know that

$$
f_{k}\left(\varepsilon^{\mu} z\right)=\varepsilon^{\mu} f_{k}(z) \text { and } f_{k}^{\prime}\left(\varepsilon^{\mu} z\right)=f_{k}^{\prime}(z)=\frac{1}{k} \sum_{\mu=0}^{k-1} f^{\prime}\left(\varepsilon^{\mu} z\right),
$$

for any $\mu=0,1, \ldots, k-1$, and summing up, we can get

$$
\begin{gathered}
\frac{1}{k} \sum_{\mu=0}^{k-1}\left[1+\frac{1}{\gamma}\left(\frac{(1-\alpha) z f^{\prime}\left(\varepsilon^{\mu} z\right)+\alpha z\left[z f^{\prime}\left(\varepsilon^{\mu} z\right)\right]^{\prime}}{(1-\alpha) f_{k}(z)+\alpha z f_{k}^{\prime}\left(\varepsilon^{\mu} z\right)}-1\right)\right] \\
=1+\frac{1}{\gamma}\left(\frac{(1-\alpha) z f_{k}^{\prime}(z)+\alpha z\left[z f_{k}^{\prime}(z)\right]^{\prime}}{(1-\alpha) f_{k}(z)+\alpha z f_{k}^{\prime}(z)}-1\right) .
\end{gathered}
$$

Hence there exist $\zeta_{\mu} \mathrm{s}$ in $\triangle$ such that

$$
1+\frac{1}{\gamma}\left(\frac{(1-\alpha) z f_{k}^{\prime}(z)+\alpha z\left[z f_{k}^{\prime}(z)\right]^{\prime}}{(1-\alpha) f_{k}(z)+\alpha z f_{k}^{\prime}(z)}-1\right) \prec \frac{1}{k} \sum_{\mu=0}^{k-1} \varphi\left(\zeta_{\mu}\right)=\varphi\left(\zeta_{0}\right),
$$

for $\zeta_{0} \in \triangle$, since $\varphi(\triangle)$ is convex. Thus $f_{k} \in \mathcal{T}_{\gamma, \alpha}(\varphi)$. 
Remark 4. Putting $\gamma=1-\lambda(0 \leq \lambda<1)$ and $\varphi(z)=\frac{1+z}{1-z}$ in the above theorem, we get the result obtained by Wang et al. [19, Lemma 3, p. 110].

Theorem 2. Let $f \in \mathcal{T}_{\gamma, \alpha}^{k}(\varphi)$ and $\operatorname{Re} \frac{1}{\alpha}[1+\alpha \gamma[\varphi(z)-1]]>0$. Then $f \in$ $\mathcal{S}_{\gamma}^{k}(\varphi)$.

Proof. Let $f \in \mathcal{T}_{\gamma, \alpha}^{k}(\varphi)$. Then by Definition 1, we have

$$
1+\frac{1}{\gamma}\left(\frac{\alpha z\left(z f^{\prime}(z)\right)^{\prime}+(1-\alpha) z f^{\prime}(z)}{\alpha z f_{k}^{\prime}(z)+(1-\alpha) f_{k}(z)}-1\right) \prec \varphi(z) .
$$

Putting $\left.p(z)=1+\frac{1}{\gamma}\left(\frac{z f^{\prime}(z)}{f_{k}(z)}\right)-1\right)$ and $\left.q(z)=1+\frac{1}{\gamma}\left(\frac{z f_{k}^{\prime}(z)}{f_{k}(z)}\right)-1\right)$, it is easy to obtain that

$$
\begin{aligned}
1+\frac{1}{\gamma} & \left(\frac{\alpha z\left(z f^{\prime}(z)\right)^{\prime}+(1-\alpha) z f^{\prime}(z)}{\alpha z f_{k}^{\prime}(z)+(1-\alpha) f_{k}(z)}-1\right) \\
& =p(z)+\frac{\alpha z p^{\prime}(z)}{1+\alpha \gamma[q(z)-1]} \prec \varphi(z), \quad(z \in \triangle) .
\end{aligned}
$$

Since $f \in \mathcal{T}_{\gamma, \alpha}^{k}(\varphi)$, then by using Theorem 1 , we can see that $q(z) \prec \varphi(z)$. Now an application of Lemma 1, yields

$$
p(z)=1+\frac{1}{\gamma}\left(\frac{z f^{\prime}(z)}{f_{k}(z)}-1\right) \prec \varphi(z) .
$$

That is $f \in \mathcal{S}_{\gamma}^{k}(\varphi)$. We thus complete the proof of Theorem 2 .

Theorem 3. Let $f \in \mathcal{S}_{\gamma}^{k}(\varphi)$ and $\operatorname{Re} \frac{1}{\alpha}[1+\alpha \gamma(\varphi(z)-1)]>0$ in $\triangle$ and let $F$ be the integral operator defined by $(2.2)$, then $F \in \mathcal{S}_{\gamma}^{k}(\varphi)$.

Proof. Let a function $f_{k}(z)$ of the form (1.2) with $F(z)$ be put in the place of $f(z)$. That is $F_{k}(z)=\frac{1}{k} \sum_{v=0}^{k-1} \varepsilon^{-v} f\left(\varepsilon^{v} z\right)$. We can see that $F_{k}(z)=$ $\frac{1}{\alpha z^{(1 / \alpha)-1}} \int_{0}^{z}\left(t^{(1 / \alpha)-2} f_{k}(t)\right) d t$ and then differentiating with respect to $z$, we get

$$
(1-\alpha) F_{k}(z)+\alpha z F_{k}^{\prime}(z)=f_{k}(z) .
$$

From (2.2), we have

$$
(1-\alpha) F(z)+\alpha z F^{\prime}(z)=f(z) .
$$

Since $f \in \mathcal{S}_{\gamma}^{k}(\varphi)$, we can apply Theorem 1 with $\alpha=0$ to deduce $f_{k} \in \mathcal{S}_{\gamma}(\varphi)$. Applying Proposition 2, we have $F_{k} \in \mathcal{S}_{\gamma}(\varphi)$, that is

$$
1+\frac{1}{\gamma}\left(\frac{z F_{k}^{\prime}(z)}{F_{k}(z)}-1\right) \prec \varphi(z) \text {. }
$$

If we denote

$$
p(z)=1+\frac{1}{\gamma}\left(\frac{z F^{\prime}(z)}{F_{k}(z)}-1\right)
$$


and

$$
q(z)=1+\frac{1}{\gamma}\left(\frac{z F_{k}^{\prime}(z)}{F_{k}(z)}-1\right),
$$

then $p(z)$ is analytic in $\triangle$ with $p(0)=1$ and $q(z)$ is analytic in $\triangle$ with $q(0)=1, q(z) \prec \varphi(z)$. Differentiating in (2.6) and using (2.8), we have

$$
\gamma p(z)-\gamma+1+\frac{\alpha \gamma z p^{\prime}(z)}{\left(1-\alpha+\alpha \frac{z F_{k}^{\prime}(z)}{F_{k}(z)}\right)}=\frac{z f^{\prime}(z)}{(1-\alpha) F_{k}(z)+\alpha z F_{k}^{\prime}(z)} .
$$

In view of (2.5) and (2.9), (2.10) gives

$$
p(z)+\frac{\alpha z p^{\prime}(z)}{1+\alpha \gamma(q(z)-1)}=1+\frac{1}{\gamma}\left(\frac{z f^{\prime}(z)}{f_{k}(z)}-1\right) \prec \varphi(z) .
$$

Now applying Lemma 1 , we get $p(z) \prec \varphi(z)$, which proves the theorem.

The method of proving Theorem 4 is similar to that of Theorem 2 .

Theorem 4. Let $\operatorname{Re} \frac{1}{\alpha}[1+\alpha \gamma[\varphi(z)-1]]>0$. Then $\mathcal{F}_{\gamma, \alpha}^{k}(\varphi) \subset \mathcal{F}_{\gamma, 0}^{k}(\varphi)$.

Now, we give the integral representations of functions belonging to the classes $\mathcal{T}_{\gamma, \alpha}^{k}(\varphi)$.

Theorem 5. Let $f \in \mathcal{T}_{\gamma, \alpha}^{k}(\varphi)$, then we have

$$
f_{k}(z)=\frac{1}{\alpha} z^{1-\frac{1}{\alpha}} \int_{0}^{z} \exp \left\{\frac{\gamma}{k} \sum_{\mu=0}^{k-1} \int_{0}^{\varepsilon^{\mu} t} \frac{\varphi(\omega(\xi))-1}{\xi} d \xi\right\} t^{\frac{1}{\alpha}-1} d t
$$

where $f_{k}(z)$ is given by equality $(1.2)$ and $\omega(z) \in \Omega$.

Proof. Suppose that $f \in \mathcal{T}_{\gamma, \alpha}^{k}(\varphi)$. We know that the condition (1.5) can be written as follows

$$
1+\frac{1}{\gamma}\left(\frac{\alpha z\left(z f^{\prime}(z)\right)^{\prime}+(1-\alpha) z f^{\prime}(z)}{\alpha z f_{k}^{\prime}(z)+(1-\alpha) f_{k}(z)}-1\right)=\varphi(\omega(z)) .
$$

By similar application of the arguments given in the proof for Theorem 1 to (2.12), we obtain

$$
1+\frac{1}{\gamma}\left(\frac{\alpha z\left(z f_{k}^{\prime}(z)\right)^{\prime}+(1-\alpha) z f_{k}^{\prime}(z)}{\alpha z f_{k}^{\prime}(z)+(1-\alpha) f_{k}(z)}-1\right)=\frac{1}{k} \sum_{\mu=0}^{k-1} \varphi\left(\omega\left(\varepsilon^{\mu} z\right)\right) .
$$

From (2.13), we have

$$
\frac{\alpha\left(z f_{k}^{\prime}(z)\right)^{\prime}+(1-\alpha) f_{k}^{\prime}(z)}{\alpha z f_{k}^{\prime}(z)+(1-\alpha) f_{k}(z)}-\frac{1}{z}=\frac{\gamma}{k} \sum_{\mu=0}^{k-1} \frac{\varphi\left(\omega\left(\varepsilon^{\mu} z\right)\right)-1}{z} .
$$


Integrating this equality, we get

$$
\log \left[\frac{\alpha z f_{k}^{\prime}(z)+(1-\alpha) f_{k}(z)}{z}\right]=\frac{\gamma}{k} \sum_{\mu=0}^{k-1} \int_{0}^{z} \frac{\varphi\left(\omega\left(\varepsilon^{\mu} \rho\right)\right)-1}{\rho} d \rho,
$$

that is,

$$
\alpha z f_{k}^{\prime}(z)+(1-\alpha) f_{k}(z)=z \exp \frac{\gamma}{k} \sum_{\mu=0}^{k-1} \int_{0}^{z} \frac{\varphi\left(\omega\left(\varepsilon^{\mu} \rho\right)\right)-1}{\rho} d \rho,
$$

or equivalently,

$$
(1-\alpha) f_{k}(z)+\alpha z f_{k}^{\prime}(z)=z \exp \frac{\gamma}{k} \sum_{\mu=0}^{k-1} \int_{0}^{\varepsilon^{\mu} z} \frac{\varphi(\omega(\zeta))-1}{\zeta} d \zeta .
$$

From (2.16), we can get equality (2.11) easily. This completes the proof of Theorem 5 .

\section{Remark 5.}

(i) Putting $\gamma=1$ in the above theorem, we get the result obtained by Wang et al. [18, Theorem 5, p. 102].

(ii) Putting $\gamma=1$ and $\alpha=1$ in the above theorem, we get the result obtained by Wang et al. [18, Theorem 3, p. 101].

(iii) For $\gamma=1$ and $\varphi(z)=\frac{1+(1-2 \lambda) z}{1-z}(0 \leq \lambda<1)$ in the above theorem, we get the result obtained by Wang et al. [19, Theorem 1, p. 112].

(iv) Putting $\gamma=1$ in our results, we get the results obtained by Paravatham and Radha [11].

\section{REFERENCES}

[1] Abubaker, A. A. A., Darus, M., On starlike and convex functions with respect to k-symmetric points, Internat. J. Math. Math. Sci., 2011 (2011), Art. ID 834064, 9 p.

[2] Al-Oboudi, F. M., On classes of functions related to starlike functions with respect to symmetric conjugate points defined by a fractional differential operator, Complex Anal. Oper. Theory 5 (2011), 647-658.

[3] Altintas, O., Irmak, H., Owa, S., Srivastava, H. M., Coefficient bounds for some families of starlike and convex functions of complex order, Appl. Math. Letters 20 (2007), 1218-1222.

[4] Chand, R., Singh, P., On certain schlicht mappings, Indian J. Pure Appl. Math. 10 (9) (1979), 1167-1174.

[5] Das, R. N., Singh, P., On subclasses of schlicht mapping, Indian J. Pure Appl. Math. 8 (1977), 864-872.

[6] Eenigenburg, P., Miller, S. S., Mocanu, P. T., Reade, M. O., On a Briot-Bouquet differential subordination, Rev. Roumaine Math. Pures Appl. 29 (1984), 567-573.

[7] Ma, W. C., Minda, D. A., A unified treatment of some special classes of univalent functions, in: Proceedings of the Conference on Complex Analysis, Z. Li, F. Ren, L. Yang, and S. Zhang (Eds.), Int. Press, 1994, 157-169.

[8] Nasr, M. A., Aouf, M. K., On convex functions of complex order, Mansoura Sci. Bull. Egypt. 9 (1982), 565-582. 
[9] Nasr, M. A., Aouf, M. K., Starlike functions of complex order, J. Natur. Sci. Math. 25 (1985), 1-12.

[10] Padmanabhan, K. S., Parvatham, R., Some applications of differential subordination, Bull. Aust. Math. Soc. 32 (3) (1985), 321-330.

[11] Parvatham, R., Radha, S., On $\alpha$-starlike and $\alpha$-close-to-convex functions with respect to n-symmetric points, Indian J. Pure Appl. Math. 16 (9) (1986), 1114-1122.

[12] Pascu, N. N., Alpha-close-to-convex functions, in: Romanian-Finnish Seminar on Complex Analysis (Bucharest, 1976), Springer, Berlin, 1979, 331-335.

[13] Pascu, N. N., Podaru, V., On the radius of alpha-starlikeness for starlike functions of order beta, in: Complex analysis - fifth Romanian-Finnish seminar, Part 1 (Bucharest, 1981), Springer, Berlin, 1983, 336-349.

[14] Ramachandran, C., Kavitha, D., Soupramanien, T., Certain bound for $q$-starlike and q-convex function with respect to symmetric points, Internat. J. Math. Math. Sci. 2015 (2015), Art. ID 205682, 7 pp.

[15] Ravichandran, V., Polatoglury, Y., Bolcaly, M., Seny, A., Certain subclasses of starlike and convex functions of complex order, Hacettepe J. Math. Stat. 34 (2005), 9-15.

[16] Robertson, M. S., On the theory of univalent functions, Ann. Math. 37 (1936), 374408.

[17] Sakaguchi, K., On certain univalent mapping, J. Math. Soc. Japan 11 (1959), 72-75.

[18] Wang, Z.-G., Gao, C.-Y., Yuan, S.-M., On certain subclasses of close-to-convex and quasi-convex functions with respect to $k$-symmetric points, J. Math. Anal. Appl. 322 (1) (2006), 97-106.

[19] Wang, Z.-G., Gao, C.-Y., Orhan, H., Akbulut, S., Some subclasses of close-to-convex and quasi-convex functions with respect to k-symmetric points, General Math., 15 (4) (2007), 107-119.

[20] Wiatrowski, P., The coefficient of a certain family of holomorphic functions, Zeszyty Nauk. Uniw. Łódz. Nauki Mat. Przyrod. Ser. II No. 39 Mat. (1971), 75-85.

[21] Yuan, S.-M., Liu, Z.-M., Some properties of $\alpha$-convex and $\alpha$-quasiconvex functions with respect to n-symmetric points, Appl. Math. Comput. 188 (2) (2007), 1142-1150.

H. E. Darwish

Department of Mathematics

Faculty of Science

Mansoura University

Mansoura, 35516

Egypt

e-mail: Darwish333@yahoo.com

S. M. Sowileh

Department of Mathematics

Faculty of Science

Mansoura University

Mansoura, 35516

Egypt

e-mail: s_soileh@yahoo.com

Received April 19, 2016

\author{
A. Y. Lashin \\ Department of Mathematics \\ Faculty of Science \\ Mansoura University \\ Mansoura, 35516 \\ Egypt \\ e-mail: aylashin@mans.edu.eg
}

